should be included. The black-body disk temperature of Jupiter then becomes $28900^{\circ} \pm$ $2700^{\circ} \mathrm{K}$.

October 21, 1965

HARRY E. HARDEBECK

Center for Radiophysics and Space Research

CORNELL UNIVERSITY

Drake, F. D., 1964, A.J., 69, 62.

Hardebeck, H. E., 1965, $A p . J, 141,837$.

Kellermann, K. I, Ap. J., 140, 969.

\title{
REFERENCES
}

\section{SPECTROGRAPHIC OBSERVATIONS OF COMET IKEYA-SEKI (1965f)}

Spectra of the "sun-grazer" comet Ikeya-Seki (1965f) have been secured at Haute Provence Observatory by astronomers from Lyon (M. Bloch), Liège (C. Arpigny, F. Dossin), and Marseille (M. Bretz).

The dispersions used are as follows: $(a)$ at the prism spectrograph of the $120-\mathrm{cm}$ telescope: $76 \AA / \mathrm{mm}$ at $\mathrm{H} \gamma, 235 \AA / \mathrm{mm}$ near $\lambda$ 5893; (b) at the grating spectrograph of the 193-cm telescope (see Fehrenbach 1960): $40 \AA / \mathrm{mm}$ (Camera II) and $4 \AA / \mathrm{mm}$ (Camera V).

On October 13, one low-dispersion and one medium-dispersion spectrogram show the $\mathrm{Na} \mathrm{D}$-lines and the $\mathrm{C}_{2}$ Swan bands superimposed on a weak continuum. $\mathrm{CN}$ is absent, although the plates have been correctly exposed in the region of the violet $(0,0)$ band.

On October 21 (10:00-11:00 U.T.) spectra obtained with the prism spectrograph show $\mathrm{Na}$ I, Ca II, and Fe I emissions, as well as a very weak reflection continuum. Between 13:00 and 14:00 U.T. on the same date a pair of 10-sec exposures (on IIa-O and on 103a-F plates) covering the spectral range from $\lambda 3580$ to $\lambda 6055 \AA$ at $4 \AA / \mathrm{mm}$ shows about eighty very sharp emission lines, mostly of neutral iron. A section of the IIa-O plate is reproduced in Figure 1.

The $\mathrm{H}$ - and $\mathrm{K}$-emissions, like the $\mathrm{Na} \mathrm{D}$-lines are rather long, their total extension being more than $2^{\prime}$ and about $50^{\prime \prime}$, respectively. On the other hand, the iron lines are rather short (less than $15^{\prime \prime}$ ).

The following multiplets of $\mathrm{Fe} I$ have been identified (the numbers in parentheses indicate the numbers of observed lines of each multiplet): 2(1), 4(11), 5(6), 15(5), 20(8), 21(12), 23(5), 36(1), 37(3), 41(4), 42(3), 43(6), 45(4), and possibly 68(1), 321(1). In addition, the presence of $\mathrm{Ca} I$ is indicated by the $\lambda 4226.728$ line, that of $\mathrm{Ni}$ I by the $\lambda 3619.392$ line. Mn I ( $\lambda \lambda 4034.490$ and 4082.944) and $\mathrm{Al} \mathrm{I}(\lambda$ 3961.523) are probably also present.

The presence of the iron lines, which were observed visually in Comet 1882 II by Copeland and Lohse (for a discussion of the identification of these lines, see Swings 1956 and Greenstein and Arpigny 1962), is thus confirmed. The other metals mentioned above, the presence of which in comets has been discussed by Greenstein and Arpigny (1962), had never been observed before.

November 1, 1965

J. DUFAY

P. SwINGS

Ch. FEHRENBACH

Haute Provence Observatory 